\title{
Cerium Oxide Nanoparticles Promote Osteoplastic Precursor Differentiation by Activating the Wnt Pathway
}

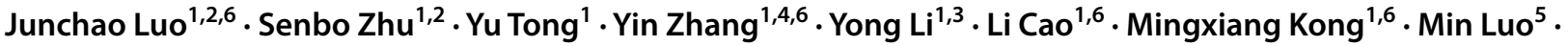 \\ Qing $\mathrm{Bi}^{1,2,6} \cdot$ Qiong Zhang ${ }^{1,6}$ (i)
}

Received: 22 December 2021 / Accepted: 16 February 2022 / Published online: 1 March 2022

(C) The Author(s) 2022

\begin{abstract}
Osteoplastic precursors are critical for fracture repair and bone homeostasis maintenance. Cerium oxide nanoparticles $\left(\mathrm{CeO}_{2} \mathrm{NPs}\right)$ can promote the osteogenic differentiation of mesenchymal stem cells and secrete vascular endothelial growth factors. However, little is known about its role in precursor osteoblasts; therefore, we further investigated the effect and mechanism of $\mathrm{CeO}_{2} \mathrm{NPs}$ in precursor osteoblasts. Cell counting kit-8 analysis was utilized to detect the toxicity of $\mathrm{CeO}_{2}$ NPs on MC3T3-E1 mouse osteogenic precursor cells. Then, alizarin red S staining was employed to assess the degree of extracellular matrix mineralization, and quantitative real-time polymerase chain reaction analysis was performed to measure the levels of osteogenesis-related genes. To identify differentially expressed genes, mRNA-sequencing was performed. Subsequently, GO and KEGG analyses were deployed to identify the major downstream pathways, whereas Western blot was used for verification. $\mathrm{CeO}_{2}$ NPs significantly enhanced the ability of MC3T3-E1 precursor osteoblasts to enhance matrix mineralization and increased the expression of osteogenic genes such as runt-related transcription factor 2, collagen I 1 , and osteocalcin. Pathway analysis revealed that $\mathrm{CeO}_{2} \mathrm{NPs}$ enhanced the nuclear translocation of $\beta$-catenin and activated the Wnt pathway by promoting family with sequence similarity 53 member B/simplet expression, while Western blot analysis indicated the same results. After using a Wnt pathway inhibitor (KYA1797K), the simulative effect of $\mathrm{CeO}_{2} \mathrm{NPs}$ was abolished. This study revealed that $\mathrm{CeO}_{2}$ NPs promoted MC3T3-E1 precursor osteoblast differentiation by activating the Wnt pathway.
\end{abstract}

Keywords Nanoparticle $\cdot$ MC3T3-E1 cells $\cdot$ Osteogenesis differentiation $\cdot$ RNA-seq

\section{Introduction}

Junchao Luo and Senbo Zhu contributed equally to this work.

Qiong Zhang

zqzjsrmyy@163.com

1 Department of Orthopedics, Zhejiang Provincial People's Hospital, Hangzhou 310014, Zhejiang, China

2 Department of Orthopedics, The Second Affiliated Hospital of Wenzhou Medical University, Wenzhou, Zhejiang, China 325027

3 Zhejiang University of Technology, Hangzhou 310014, Zhejiang, China

4 Bengbu Medical College, Bengbu 233030, Anhui, China

5 Dongyang Hospital of Traditional Chinese Medicine, Jinghua 322199, Zhejiang, China

6 Institute of Sports Medicine and Osteoarthropathy of Hangzhou Medical College, 481\# Binwen Road, Hangzhou 310000, Zhejiang, China
Osteoblast precursors play a significant role in fracture repair and bone homeostasis maintenance. Maes et al. disclosed that osteoblast precursors and vascular endothelial cells showed co-invasion during fracture healing in mice, indicating that osteoblast precursors are involved in fracture healing [1]. Age-related bone loss is intimately linked to imbalances in bone formation and resorption [2]. Mesenchymal stem cells, distributed in bone marrow and other body parts, follow the blood circulation to reach the bone surface and differentiate into precursors osteoblasts, which further form mature osteoblasts, produce bone matrix, and promote matrix mineralization [3]. Therefore, reduced differentiation of precursor osteoblasts decreased bone formation, leading to osteoporosis. Promoting differentiation of precursor osteoblasts is an important goal for fracture repair and osteoporosis treatment. 
Nanoparticles are considered promising in diagnosing and treating diseases. Passive nanoparticles (NPs) have recently been approved by the US Food and Drug Administration (FDA) for the clinical treatment of cancer [4, 5]. Kalyanaraman et al. evaluated the safety of cerium oxide nanoparticles $\left(\mathrm{CeO}_{2} \mathrm{NPs}\right.$ ) by implanting $250-1000 \mathrm{mg} \mathrm{CeO} \mathrm{NPs}_{2}$ subcutaneously in rats and observing changes in local tissues and major organs after 28 days [6]. The results demonstrated that $\mathrm{CeO}_{2}$ NPs did not significantly stimulate local tissues and showed no toxicity to major organs, implying that cerium oxide nanoparticles have good biosafety. Previous studies have demonstrated that $\mathrm{CeO}_{2} \mathrm{NPs}$ are characterized by excellent scavenging ability of active free radicals, mainly based on their potential to simulate glutathione peroxidase and catalase during the transformation of $\mathrm{Ce}^{3+}$ and $\mathrm{Ce}^{4+}$ valence states [7]. $\mathrm{Li}$ et al. found that $\mathrm{CeO}_{2} \mathrm{NPs}$ reversed the decrease of superoxide dismutase activity in $\mathrm{H}_{2} \mathrm{O}_{2}$-induced osteoblast oxidative damage and reduced the production of reactive oxygen species and malondialdehyde formation [8]. Wei et al. suggested that $\mathrm{CeO}_{2}$ NPs can induce M2-type differentiation of macrophages and promote osteogenic differentiation of mesenchymal stem cells [9]. Similarly, Purohit et al. stated that composite scaffolds with $\mathrm{CeO}_{2}$ NPs significantly promoted bone repair [10]. However, the mechanism by which $\mathrm{CeO}_{2} \mathrm{NPs}$ promote osteoblast differentiation has not been fully elucidated.

In this study, we determined the effects of $\mathrm{CeO}_{2} \mathrm{NPs}$ on MC3T3-E1 mouse precursor osteoblasts. It was revealed that $\mathrm{CeO}_{2}$ NPs promote the nuclear translocation of $\beta$-catenin protein, activate the Wnt pathway, and promote the differentiation of precursor osteoblasts by increasing Fam53B expression.

\section{Material and Methods}

\section{Preparation and Characterization of Cerium Oxide Nanoparticles}

Cerium nitrate hexahydrate was added to a mixture of ethylene glycol and double-distilled water and heated to $60{ }^{\circ} \mathrm{C}$. After adding ammonia, the solution was then agitated until it turned yellowish-white. $\mathrm{CeO}_{2}$ NPs were obtained by highspeed centrifugation. After washing three times, sodium citrate was employed as a surfactant to activate NPs. Dynamic light scattering (DLS) analysis was utilized to evaluate particle size and zeta potential. Transmission electron microscopy (TEM) was used to observe the morphology of $\mathrm{CeO}_{2}$ NPs. X-ray powder diffraction patterns were used to evaluate the crystallinity of NPs. X-ray photoelectron spectroscopy was utilized to assess the chemical composition of the surface and valence state of $\mathrm{CeO}_{2} \mathrm{NPs}$.

\section{Cell Culture}

MC3T3-E1 mouse precursor osteoblasts cells (American Type Culture Collection) were cultured with Dulbecco's Modified Eagle Medium (HyClone) supplemented with $10 \%$ fetal bovine serum, $100 \mathrm{IU} / \mathrm{mL}$ penicillin, and $100 \mu \mathrm{g} /$ $\mathrm{mL}$ streptomycin (Invitrogen) at $37{ }^{\circ} \mathrm{C}$ with $5 \% \mathrm{CO}_{2}$. The medium was replaced after 2-3 days.

\section{Cytotoxicity Test}

Cytotoxicity tests were utilized to find suitable concentrations of $\mathrm{CeO}_{2}$ NPs. MC3T3-E1 precursor osteoblasts were inoculated into 96 -well plates at a density of $5 \times 10^{3}$ cells/ well, and $\mathrm{CeO}_{2} \mathrm{NPs}$ at different concentrations $(0,1,3,10$, $30,50,100,150$, and $200 \mu \mathrm{g} / \mathrm{mL}$ ) at $37{ }^{\circ} \mathrm{C}$ for 24 and $48 \mathrm{~h}$, respectively. After discarding the medium, it is washed once with PBS. The absorbance value at $450 \mathrm{~nm}$ was detected after incubating with non-haematine medium containing $10 \%$ cell counting kit- 8 reagent (Dojindo) at $37{ }^{\circ} \mathrm{C}$ for $2 \mathrm{~h}$ and $30 \mathrm{~min}$.

\section{Alizarin Red S Staining}

The impact of $\mathrm{CeO}_{2}$ NPs on the mineralization of MC3T3E1 precursor osteoblasts was evaluated using alizarin red $\mathrm{S}$ staining. After covering the 12-well plates with gelatin, precursor osteoblasts were seeded into the plates at a density of $3 \times 10^{4}$ cells $/ \mathrm{mL}$. Precursor osteoblasts were cultured in osteogenic differentiation induction media containing $\mathrm{CeO}_{2}$ NPs at different concentrations $(0,1$, and $3 \mu \mathrm{g} / \mathrm{mL})$. After 14 days of differentiation, precursor osteoblasts were fixed with $4 \%$ paraformaldehyde phosphate buffer for $15 \mathrm{~min}$. We washed the cells with $\mathrm{ddH}_{2} \mathrm{O}$ and stained them with alizarin red S (Sigma) for $3 \mathrm{~min}$. The image was captured after the sample was rinsed twice. The procedures were independently repeated three times to ensure reliability.

\section{Quantitative Real-Time Polymerase Chain Reaction Analysis}

The effect of $\mathrm{CeO}_{2}$ NPs on osteogenesis differentiation of precursor osteoblasts was further analyzed by quantitative real-time polymerase chain reaction analysis (qRT-PCR) according to the published protocol [11]. We used RNAquick purification kit (ESscience) to extract total cellular RNA. The cDNA was synthesized using $1 \mu \mathrm{g}$ of RNA utilizing a PrimescriptTM RT reagent kit with a gDNA eraser (Takara Bioindustry) according to manufacturer's protocol. cDNA was diluted with nuclease-free water in a ratio of 1:10. Maxima SYBR green PCR master mix $(2 \times)$ was used 
for real-time PCR analysis. The program used for real-time PCR included initial denaturation at $95{ }^{\circ} \mathrm{C}$ for $2 \mathrm{~min}$, followed by 40 cycles of denaturation at $95{ }^{\circ} \mathrm{C}$ for $15 \mathrm{~s}$, annealing at $60{ }^{\circ} \mathrm{C}$ for $30 \mathrm{~s}$ and extension at $72{ }^{\circ} \mathrm{C}$ for $30 \mathrm{~s}$. Target gene expression was normalized to GAPDH expression, and $2^{-\Delta \Delta C T}$ method was utilized to determine relative gene expression. The primers of runt related transcription factor 2 (Runx2), osteocalcin (OCN), collagen I $\alpha 1$ (Col $\mathrm{I} \alpha 1)$, family with sequence similarity 53 member B (Fam53B), and GAPDH were designed with the aid of NCBI Primer-Blast Tool, which were listed as follows: Runx2 (F) 5'-AGAGTC AGATTACAGATCCCAGG-3', (R) 5'-TGGCTCTTCTTA CTGAGAGAGG-3'; OCN (F) 5'-TAGTGAACAGACTCC GGCGCTA-3', (R) 5'-TGTAGGCGGTCTTCAAGCCAT3'; Col I 1 (F) 5'-GCTTCACCTACAGCACCCTTGT-3', (R) 5'-TGACTGTCTTGCCCCAAGTTC-3'; Fam53B (F) 5'-ACCTGGATAGAAAATGCCCTCT-3', (R)5'-GAGGTC TTTGATTAGGCTGGTC-3'; GAPDH (F) 5'-TGACCTCAA CTACATGGTCTACA-3', (R) 5'-CTTCCCATTCTCGGC CTTG-3'.

\section{mRNA-Sequencing}

Total cellular RNA was extracted following the same aforementioned method. Messenger (m)RNA for each sample was enriched using poly-T oligo-attached magnetic beads. The first strand cDNA was synthesized using random hexamer primer and RNase H. Second strand cDNA synthesis was subsequently performed using buffer, dNTPs, DNA polymerase I, and RNase $\mathrm{H}$. The purified cDNA was subjected to a final reparation, the addition of an A-tailing, and an adapter. Ultimately, cDNA library was sequenced on Illumina Hiseq xTen platform.

\section{Western Blot Analysis}

Western blotting analysis was performed as described previously [12]. Total protein was extracted by direct lysis of cells using cold RIPA lysis buffer containing $1 \mathrm{mmol} / \mathrm{L}$ phenylmethylsulfonyl fluoride. Nuclear protein was extracted using a nuclear protein extraction kit (Solarbio) following manufacturer's protocol. The protein concentration was determined using a Pierce ${ }^{\mathrm{TM}} \mathrm{BCA}$ protein assay kit (Thermo Scientific). Equal amounts of protein $(20 \mu \mathrm{g})$ were fractionated on precast protein improve gels (Yeasen Biotechnology) and transferred to a PVDF membrane (Merck Millipore). The membrane was then blocked with $5 \%$ nonfat dry milk at room temperature for $2 \mathrm{~h}$. After three additional washes with TBST, membranes were incubated overnight with primary antibodies at $4{ }^{\circ} \mathrm{C}$. The primary antibodies are as follows: Runx 2 (mouse mAb; cat. no. ab76956; Abcam), OCN (rabbit mAb; cat. no. ab93876; Abcam), Fam53B (rabbit mAb; cat. no. NBP1-88,976; Novus), GAPDH (mouse mAb; cat. no. ab8245; Abcam), $\beta$-catenin (rabbit mAb; cat. no. 51067-2-AP; Proteintech), lamin A/C (rabbit mAb; cat. no. 51067-2-AP; Proteintech). After washing three times with TBST for $10 \mathrm{~min}$, membranes were then incubated with HRP-conjugated secondary antibodies (goat AntiRabbit IgG, Cat.no.ZB-2301, Zhongshan Golden Bridge Biotechnology; goat Anti-Mouse IgG, cat. no. A0216, Beyotime Institute of Biotechnology) for $2 \mathrm{~h}$ at room temperature. After being washed three more times with TBST, protein bands were visualized using an ECL kit (FDbio Science Biotechnology).

\section{Statistical Analysis}

All data are presented as mean \pm standard deviation (SD). The data comparisons between multiple groups were analyzed using one-way analysis of variance (ANOVA), whereas comparisons between two groups were analyzed using Student's $t$-test. A $p$-value less than 0.05 was considered significant. During the differential expression analysis, all $p$-values were adjusted using Benjamini and Hochberg method. Genes with $q \leq 0.05$ and $\mid \log _{2}$ Ratiol $\geq 1$ are identified as differentially expressed genes (DEGs). GO enrichment of DEGs was implemented by the hypergeometric test, in which $p$-value is calculated and adjusted as a $q$-value. GO terms with $q<0.05$ were considered significantly enriched. KEGG enrichment of DEGs was performed using hypergeometric test, in which $p$-value was adjusted by multiple comparisons as $q$-value, which was considered significantly enriched when $p$-value was less than 0.05. Differential expression analysis was performed using DEGseq R package (v1.16), and other analyses were performed using SPSS program version 23.0 (IBM Corp., Armonk, NY).

\section{Results}

\section{Characterization of Cerium Oxide Nanoparticles}

The results of DLS revealed that the mean particle size and zeta potential of $\mathrm{CeO}_{2} \mathrm{NPs}$ were $12.13 \mathrm{~nm}$ and $-36 \mathrm{mV}$, respectively (Fig. 1a-b). TEM demonstrated that most nanoparticles were oval in shape (Fig. 1c). The diffraction peaks from powder X-ray diffraction patterns were well matched to the standard cerium oxide pattern (PDF2:34-0394), indicating the high purity of our synthetic nanoparticles (Fig. 1d). X-ray photoelectron spectroscopy revealed that the relative levels of $\mathrm{Ce}^{4+}$ and $\mathrm{Ce}^{3+}$ were $42.13 \%$ and $57.87 \%$ (Fig. 1e-f). 

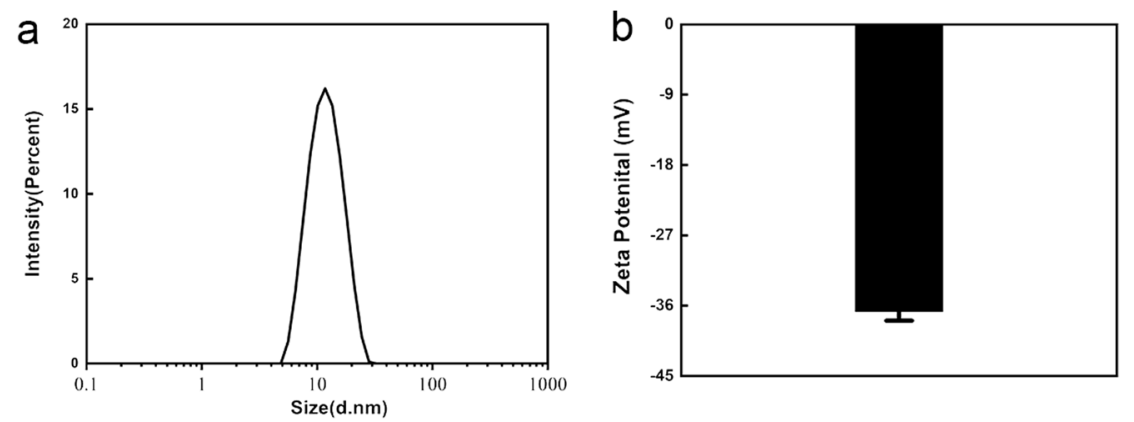

C
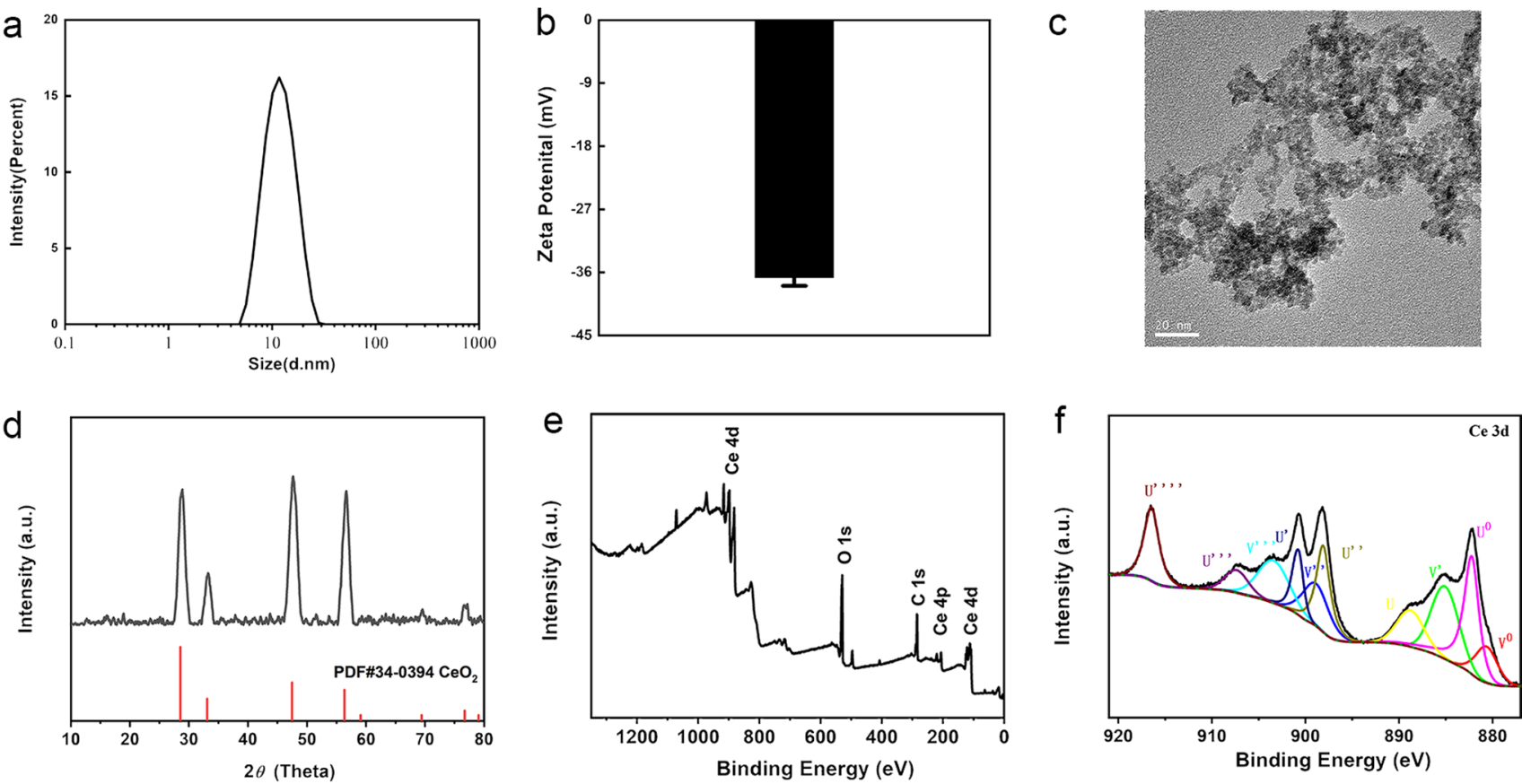

Fig. 1 Characterization of cerium oxide nanoparticles. A Particle size distribution. B Zeta potential. C A transmission electron microscopy image. D X-ray powder diffraction patterns for $\mathrm{CeO}_{2} \mathrm{NPs}$. E X-ray

\section{Cerium Oxide Nanoparticles Promoted Precursor Osteoblast Differentiation in MC3T3-E1 Cells}

CCK-8 analysis indicated that $\mathrm{CeO}_{2}$ NPs had a non-significant effect on chondrocyte viability in the range of $0-3 \mu \mathrm{g} /$ $\mathrm{mL}$ (Fig. 2a). Consequently, we used concentrations of 1 and $3 \mu \mathrm{g} / \mathrm{mL}$ NPs for subsequent in vitro experiments. Then, Alizarin red staining and qPCR were utilized to determine the effect of $\mathrm{CeO}_{2}$ NPs on osteogenic differentiation of MC3T3-E1 cells. As displayed in Fig. 2b-c, we detected a significant increase in $\mathrm{Ca}^{2+}$ accumulation in the extracellular matrix. Among them, $\mathrm{CeO}_{2} \mathrm{NP}-$ mineralized nodules at a $1 \mu \mathrm{g} / \mathrm{ml}$ concentration were the most significant. Additionally, we conducted qPCR to examine the expression of osteogenic marker genes (Runx2, Col I $\alpha 1$ and OCN) in MC3T3-E1 cells and the effect of $\mathrm{CeO}_{2}$ NPs on enhancing osteogenesis. Compared with the control group, MC3T3-E1 cells treated with $\mathrm{CeO}_{2}$ NPs for 48 h showed significantly enhanced osteogenic differentiation (Fig. 2d).

\section{Functional Annotation and Pathways Analysis of Differential Expressed Genes}

Considering that $1 \mu \mathrm{g} / \mathrm{mL} \mathrm{CeO}_{2}$ NPs demonstrated the best effect, we used this concentration in subsequent experiments. MC3T3-31 mouse precursor osteoblasts were treated with $1 \mu \mathrm{g} / \mathrm{mL}$ NPs for $48 \mathrm{~h}$, and RNA of it and control group were extracted for sequencing. The results revealed that photoelectron spectroscopy spectra of $\mathrm{CeO}_{2}$ NPs. F Development of Ce 3d X-ray photoelectron spectroscopy spectra and fitted Ce 3d $\mathrm{X}$-ray photoelectron spectroscopy spectra

seven DEGs were upregulated, and four were downregulated compared with the control group. Heatmap indicated that DEGs had similar expression patterns within groups and different expression patterns between groups (Fig. 3a). These DEGs were significantly enriched in 30 GO items, such as negative regulation of nitric-oxide synthase activity in biological process and Wnt signalosome in cell composition $(q<0.05)$ (Fig. 3b). Furthermore, 20 KEGG pathways were substantially enriched, including nitrogen metabolism, prolactin signaling pathway, growth hormone synthesis, Wnt pathway, insulin signaling pathway, and thyroid hormone signaling pathway (Fig. 3c).

Wnt pathway activation is critical in differentiating precursor osteoblasts. As depicted in Fig. 3a, Fam53B gene expression was substantially upregulated in MC3T3-E1 cells treated with $\mathrm{CeO}_{2}$ NPs. The Simplet/Fam53B belongs to a family of proteins (Fam53A, Fam53B, and Fam53C), and previous studies have revealed its involvement in Wnt pathway transduction by promoting $\beta$-catenin nuclear translocation [13].

\section{Fam53B Activates the Wnt Pathway by Promoting Beta-Catenin Nuclear Translocation}

To determine whether Fam53B gene expression was increased, quantitative real-time polymerase chain reaction (qRTPCR) analysis and Western blot (WB) analysis were used for verification. After treating MC3T3-E1 mouse precursor 
Fig. 2 Cerium oxide nanoparticles increased osteogenic differentiation of MC3T3-E1 precursor osteoblast. A Effect of cerium oxide nanoparticles on cell viability of MC3T3-E1 cells. B Alizarin red S staining was performed on days 14 after treatment with cerium oxide nanoparticles. C Semiquantitative analysis of calcium deposits. D qRT-PCR was employed to examine changes in the levels of Runx2, osteocalcin, and collagen I $\alpha 1$ in MC3T3-E1 cells after treatment with cerium oxide nanoparticles for $48 \mathrm{~h}$ a

$24 \mathrm{~h}$

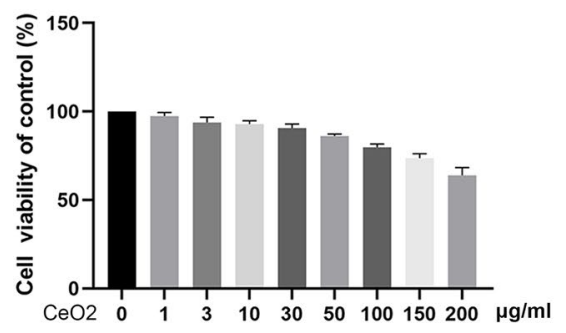

b

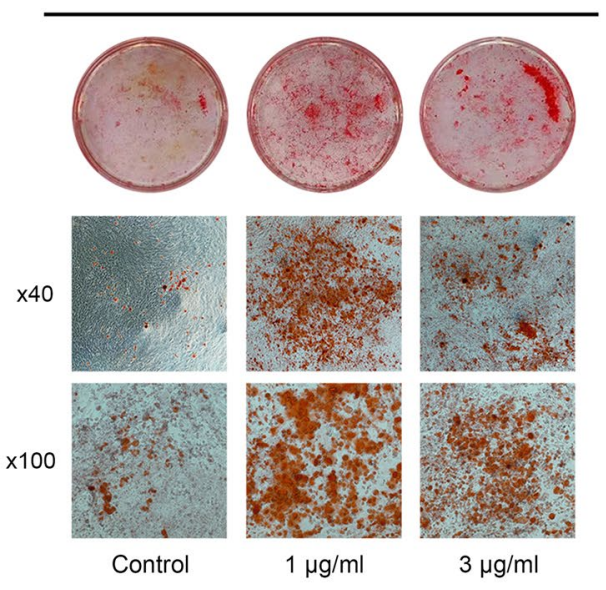

c

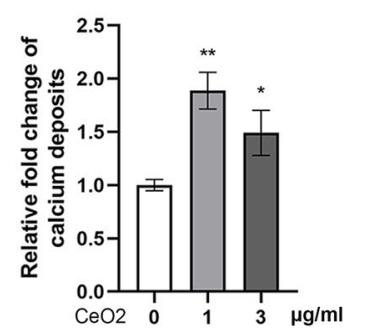

$48 \mathrm{~h}$

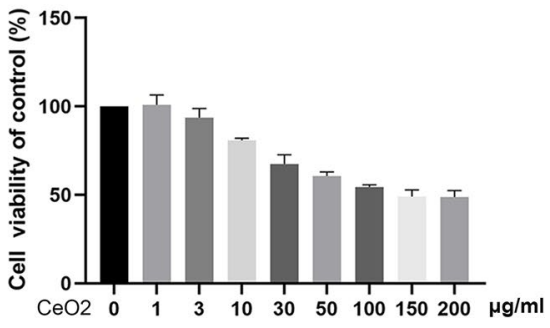

d Runx2

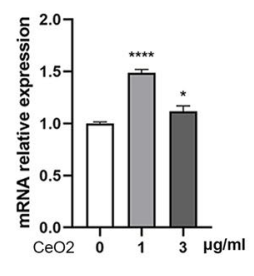

OCN

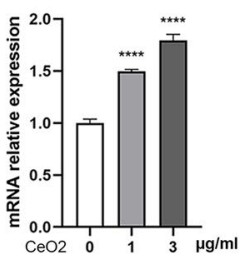

Collage I

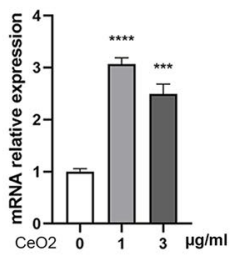

osteoblasts with $1 \mu \mathrm{g} / \mathrm{mL}$ cerium oxide nanoparticles for 48 h, we observed a significant increase in Fam53B expression (Fig. 4a). Subsequently, we determined whether Fam53B bridged $\mathrm{CeO}_{2}$ NPs and $\beta$-catenin signaling. We treated MC3T3-E1 cells with different combinations of $\beta$-catenin inhibitor (KYA1797K) and $\mathrm{CeO}_{2}$ NPs for $48 \mathrm{~h}$. As expected, Fam53B significantly increased the nuclear accumulation of $\beta$-catenin and the expression of downstream osteogenic genes OCN and Runx2. KYA1797K partially reversed the effect of $\mathrm{CeO}_{2}$ NPs on osteogenic differentiation (Fig. 4b-d).

\section{Discussion}

The role of $\mathrm{CeO}_{2}$ NPs in osteogenic differentiation has been partially demonstrated in previous studies. Lu et al. disclosed that $\mathrm{CeO}_{2}$ NPs promote osteogenic differentiation of bone marrow mesenchymal stem cells by activating ERK pathway [14]. Vascular invasion is the premise of bone remodeling and fracture repair. Xiang et al. stated that $\mathrm{CeO}_{2}$ NPs promote proliferation, differentiation, and angiogenesis of endothelial progenitor cells by increasing 


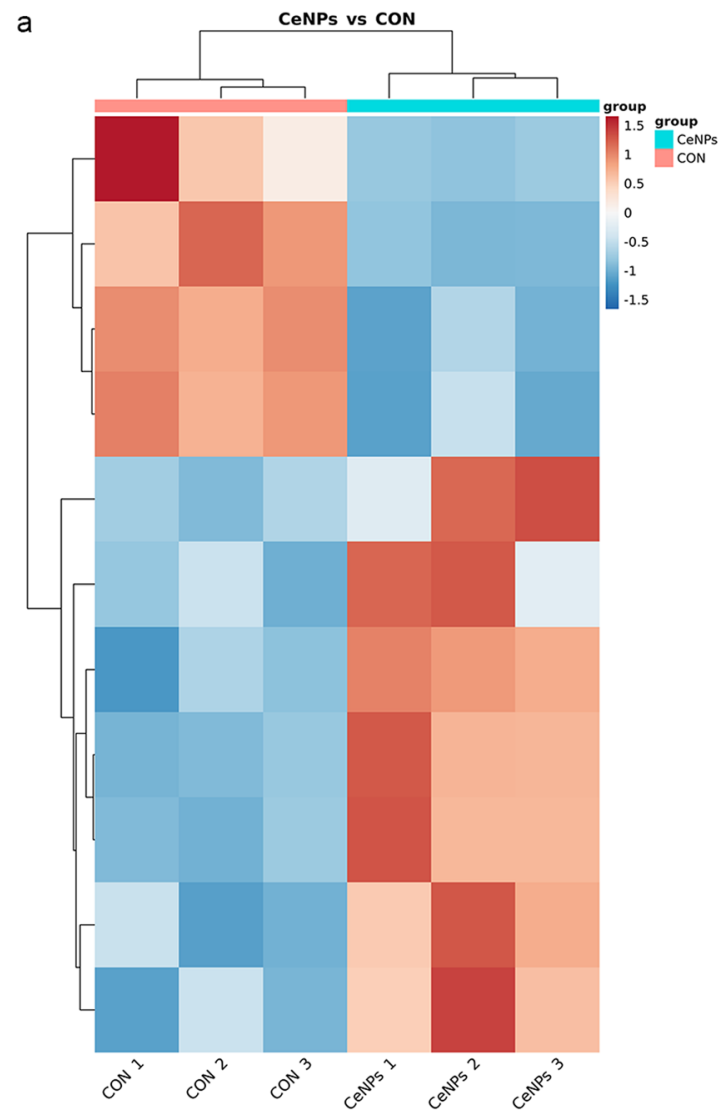

b

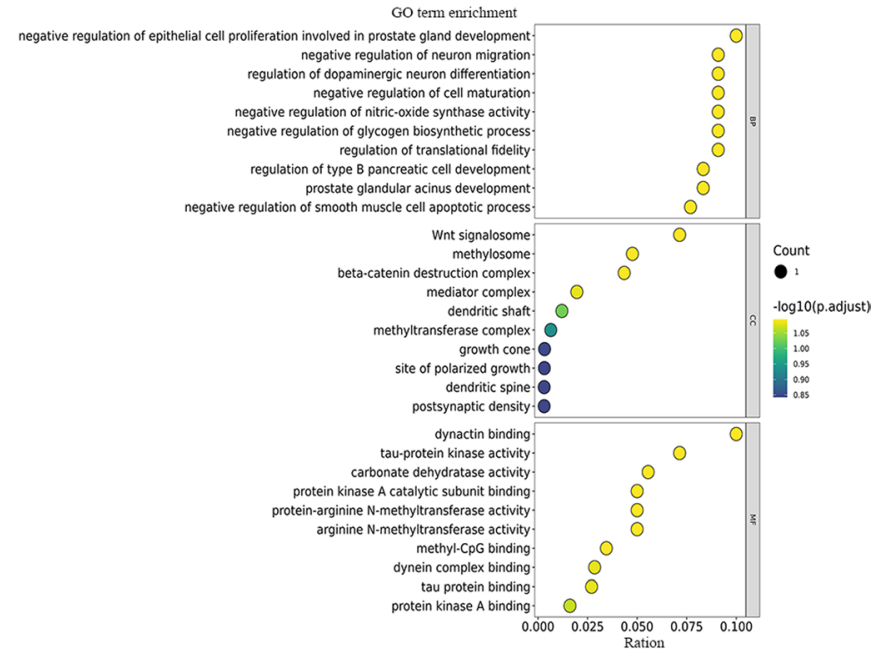

c

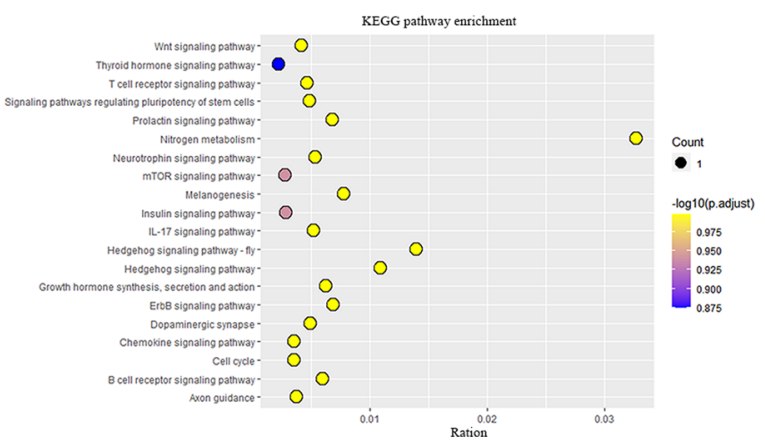

Fig. 3 Heatmap and enrichment analysis of cerium oxide nanoparticles induced by differential gene expression. A Heatmap. B GO term enrichment. c KEGG pathway enrichment

the stability of HIF-1 1 in mesenchymal stem cells and increasing vascular endothelial growth factor expression [15]. However, the mechanism of $\mathrm{CeO}_{2} \mathrm{NPs}$ in precursor osteoblasts has not been elucidated.

Although no human pharmacokinetic studies have been performed, CCK-8 analysis suggested that $0-3 \mu \mathrm{g} /$ $\mathrm{mL} \mathrm{CeO}{ }_{2}$ NPs had no significant effect on cell viability of MC3T3-E1 precursor osteoblasts. The influence of 1 and $3 \mu \mathrm{g} / \mathrm{mL} \mathrm{CeO}_{2}$ NPs on promoting osteogenesis and its mechanism were further investigated in subsequent experiments. Alizarin Red S staining was performed first. Extracellular matrix mineralization is a major part of bone formation; consequently, the number and size of calcium nodules can be used to measure the degree of osteoblast differentiation. The results demonstrated that $\mathrm{CeO}_{2}$ NPs significantly enhanced the extracellular matrix mineralization of MC3T3-E1 precursor osteoblasts, especially at $1 \mu \mathrm{g} / \mathrm{mL}$. Runx 2 and osteocalcin are significant marker genes for osteogenic differentiation, and their expression is increased at different osteogenic differentiation stages $[16,17]$. Col I $\alpha 1$ is a major component of bone matrix and is secreted by osteoblasts. Our study demonstrates that $\mathrm{CeO}_{2}$ NPs significantly upregulated their expression.

Based on previously mentioned results, we suggested that $1 \mu \mathrm{g} / \mathrm{mL} \mathrm{CeO}_{2}$ NPs had the best effect. Therefore, it was used to treat MC3T3-E1 precursor osteoblasts, and total RNA was extracted for mRNA-seq. The results demonstrated that Fam53B gene expression in MC3T3-E1 precursor osteoblasts significantly increased after $\mathrm{CeO}_{2}$ NPs. After treating MC3T3-E1 mouse precursor osteoblasts with $\mathrm{CeO}_{2} \mathrm{NPs}$, we detected a significant increase in Fam53B expression. Thermes et al. found that the absence of Fam53B reduced cell proliferation in medaka fish [18]. Kizil et al. found that Fam53B loss in zebrafish cells reduced nuclear accumulation of $\beta$-catenin, a major protein downstream of Wnt pathway, while Fam53B overexpression promoted the nuclear translocation of $\beta$-catenin [13]. The bone morphogenetic protein, Wnt, Indian hedgehog, and other proteins are involved in differentiating precursor osteoblasts $[19,20]$. The $\mathrm{Wnt} / \beta$ catenin signaling pathway regulates osteogenic differentiation, bone formation, and the occurrence of various bone metabolic diseases [21]. The canonical Wnt pathway 
a

Fam53B

Fam53B
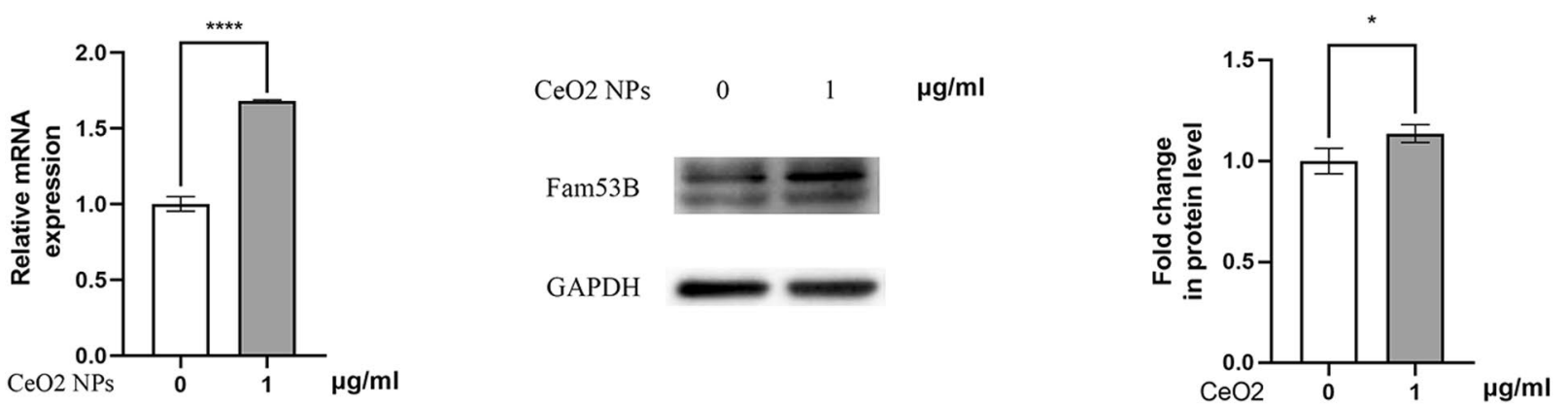

b

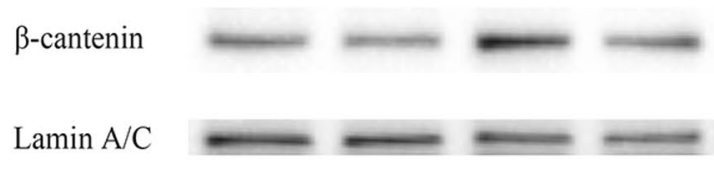

$\beta$-cantenin

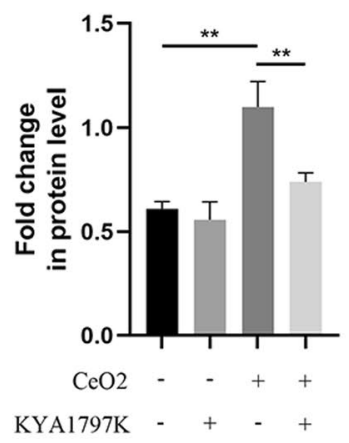

d

Osteocalcin

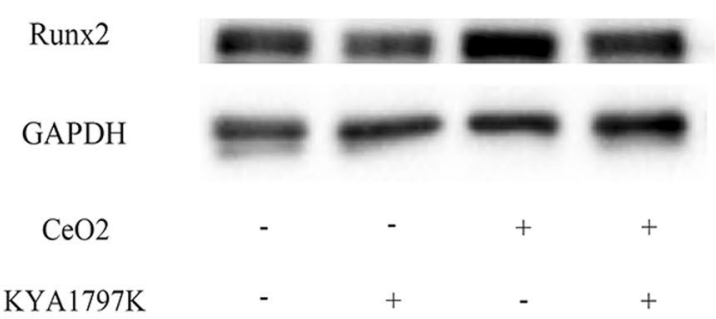

Fig. 4 Cerium oxide nanoparticles activated Wnt/ $\beta$-catenin signaling to promote osteogenesis differentiation by increasing Fam53B expression. A Fam53B expression in MC3T3-E1 treated with cerium oxide nanoparticles for $48 \mathrm{~h}$ using qRT-PCR, Western blotting, and quantification related to GAPDH levels. B The levels of $\beta$-catenin protein in the nucleus of MC3T3-E1 treated with $1 \mu \mathrm{g} / \mathrm{mL}$ cerium oxide nano-
C

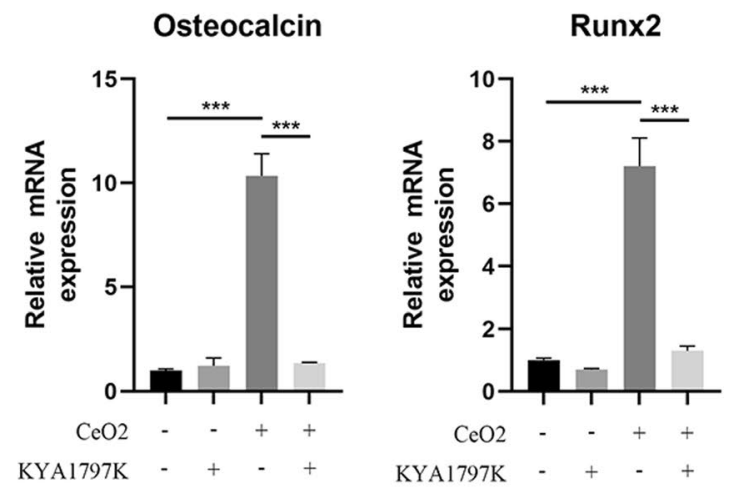

e

Osteocalcin

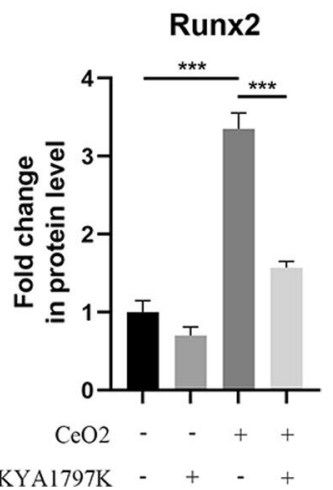

particles in the presence or absence of KYA1797K after 48 h. C qRTPCR was used to examine changes in the levels of Runx2, osteocalcin in MC3T3-E1 treated with cerium oxide nanoparticles in the presence or absence of KYA1797K after 48 h. D Representative Western blot images of Runx2 and osteocalcin in MC3T3-E1 treated as described above. E Quantitative analysis of Runx 2 and osteocalcin 


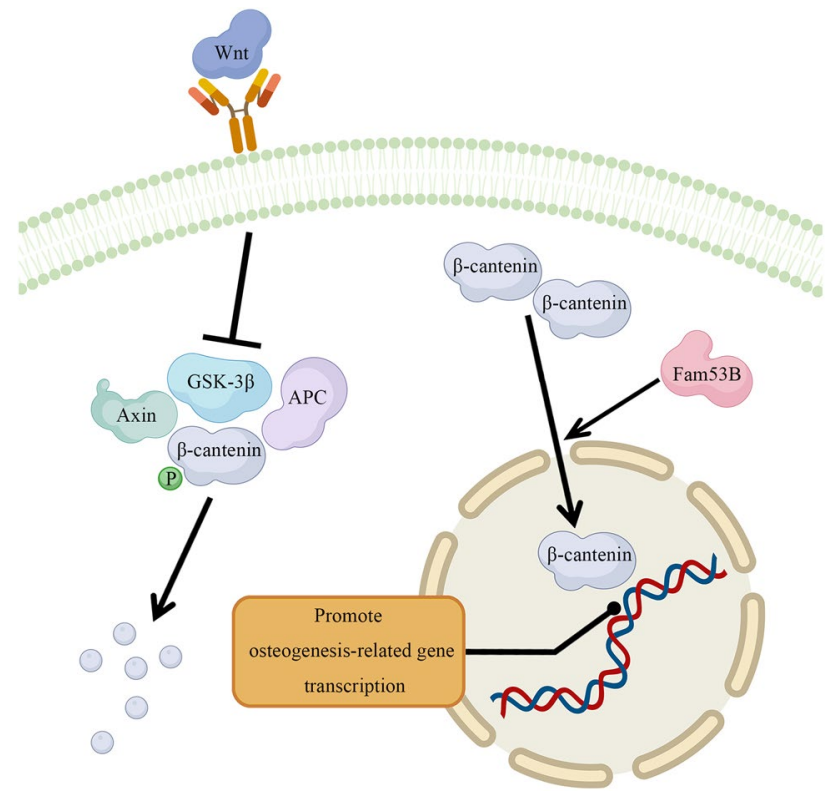

Fig. 5 Potential mechanism of cerium oxide nanoparticles promoting osteogenesis. $\beta$-catenin proteins mediate the canonical Wnt pathway. In the cytoplasm, $\beta$-catenin is phosphorylated by the complexes of GSK-3 $\beta$, APC, and Axin and is subsequently degraded. Wnt stimulation inhibited GSK-3 $\beta$ activity, resulting in $\beta$-catenin accumulation in the cytoplasm, which translocates into the nucleus later and promotes the transcription of target genes. Cerium oxide nanoparticles promoted $\beta$-catenin nuclear translocation by promoting Fam53B protein expression

is initiated by binding Frizzled transmembrane receptors, low-density lipoprotein receptor-related protein 5 (LRP5), and/or LRP6 bind to secreted Wnts, resulting in activated dishevelled proteins [22]. Subsequently, activated dishevelled phosphorylates glycogen synthase kinase-3 $\beta$ (GSK-3 $\beta$ ), inhibiting its function. $\beta$-catenin protein was released from the complex of GSK-3 $\beta$, adenomatous polyposis coli (APC), and axin. Then, $\beta$-catenin protein accumulates in the cytoplasm and translocates into the nucleus. Using $\mathrm{T}$ cell factor/lymphoid enhancing factor (TCF/LEF), $\beta$-catenin can activate the expression of c-myc, c-Jun, and other downstream transcription factors, thus promoting the expression of osteogenic genes [23]. In addition, $\beta$-catenin increased Runx 2 expression (Fig. 5). KYA1797K can promote $\beta$-catenin degradation. After adding KYA1797K to the culture medium, we found that promoting MC3T3-E1 precursor osteoblasts differentiation by $\mathrm{CeO}_{2}$ NPs was abolished.

This study also has certain limitations. We only evaluated the effect of $\mathrm{CeO}_{2} \mathrm{NPs}$ in vitro, lacking data in vivo experiments. Therefore, in future studies, we will further evaluate the role of $\mathrm{CeO}_{2}$ NPs in vivo.

\section{Conclusion}

To summarize, $\mathrm{CeO}_{2}$ NPs promoted Fam53B expression, which increased the nuclear accumulation of $\beta$-catenin, thus activating Wnt pathway. Wnt pathway activation contributes to increased expression of downstream osteoblast-related genes, promoting the differentiation of MC3T3-E1 precursor osteoblasts.

Author Contribution Junchao Luo and Yu Tong conceived the project and designed the experiment. Junchao Luo, Senbo Zhu, Yin Zhang, and Li Cao performed the key experiments and prepare the manuscript. Mingxiang Kong, Yong Li, and Min Luo analyzed the data and composited the diagram. Qing Bi and Qiong Zhang directed the experiment and revised the manuscript. All authors read and approved the final version of the manuscript and all data were generated in-house and that no paper mill was used.

Funding This work was supported by the Zhejiang Province Traditional Chinese Medicine Scientific Research Fund project (2019ZB138), Medical Health Science and Technology Project of Zhejiang Provincial Health Commission (2021KY028), Basic public welfare research plan of Zhejiang Province (GF19H060034), Health Technology Project of Zhejiang Province (2020KY029) and National Science Foundation of China (Grant No. 81672769), and Basic public welfare research plan of Zhejiang Province (LGF22H060029).

Data Availability Not applicable.

\section{Declarations}

Ethics Approval This paper complies with ethical standards and does not require ethical approval.

Consent for Publication All authors agree to publish this paper.

Conflict of Interest The authors declare no competing interests.

Open Access This article is licensed under a Creative Commons Attribution 4.0 International License, which permits use, sharing, adaptation, distribution and reproduction in any medium or format, as long as you give appropriate credit to the original author(s) and the source, provide a link to the Creative Commons licence, and indicate if changes were made. The images or other third party material in this article are included in the article's Creative Commons licence, unless indicated otherwise in a credit line to the material. If material is not included in the article's Creative Commons licence and your intended use is not permitted by statutory regulation or exceeds the permitted use, you will need to obtain permission directly from the copyright holder. To view a copy of this licence, visit http://creativecommons.org/licenses/by/4.0/.

\section{References}

1. Maes C, Kobayashi T, Selig MK, Torrekens S, Roth SI, Mackem S, Carmeliet G, Kronenberg HM (2010) Osteoblast precursors, but not mature osteoblasts, move into developing and fractured 
bones along with invading blood vessels. Dev Cell 19(2):329344. https://doi.org/10.1016/j.devcel.2010.07.010

2. Kim JM, Lin C, Stavre Z, Greenblatt MB and Shim JH (2020) Osteoblast-osteoclast communication and bone homeostasis. Cells 9(9). https://doi.org/10.3390/cells9092073

3. Blair HC, Larrouture QC, Li Y, Lin H, Beer-Stoltz D, Liu L, Tuan RS, Robinson LJ, Schlesinger PH, Nelson DJ (2017) Osteoblast differentiation and bone matrix formation in vivo and in vitro. Tissue Eng Part B Rev 23(3):268-280. https://doi. org/10.1089/ten.TEB.2016.0454

4. Amreddy N, Babu A, Muralidharan R, Panneerselvam J, Srivastava A, Ahmed R, Mehta M, Munshi A, Ramesh R (2018) Recent advances in nanoparticle-based cancer drug and gene delivery. Adv Cancer Res 137:115-170. https://doi.org/10.1016/ bs.acr.2017.11.003

5. Alavi M and Hamidi M (2019) Passive and active targeting in cancer therapy by liposomes and lipid nanoparticles. Drug Metab Pers Ther 34(1). https://doi.org/10.1515/dmpt-2018-0032

6. Kalyanaraman V, Naveen SV, Mohana N, Balaje RM, Navaneethakrishnan KR, Brabu B, Murugan SS, Kumaravel TS (2019) Biocompatibility studies on cerium oxide nanoparticles - combined study for local effects, systemic toxicity and genotoxicity via implantation route. Toxicol Res (Camb) 8(1):25-37. https:// doi.org/10.1039/c8tx00248g

7. Luo J, Zhang Y, Zhu S, Tong Y, Ji L, Zhang W, Zhang Q, Bi Q (2021) The application prospect of metal/metal oxide nanoparticles in the treatment of osteoarthritis. Naunyn Schmiedebergs Arch Pharmacol 394(10):1991-2002. https://doi.org/10.1007/ s00210-021-02131-0

8. Li K, Xie Y, You M, Huang L, Zheng X (2016) Plasma sprayed cerium oxide coating inhibits $\mathrm{H} 2 \mathrm{O} 2$-induced oxidative stress and supports cell viability. J Mater Sci Mater Med 27(6):100. https:// doi.org/10.1007/s10856-016-5710-9

9. Wei F, Neal CJ, Sakthivel TS, Kean T, Seal S, Coathup MJ (2021) Multi-functional cerium oxide nanoparticles regulate inflammation and enhance osteogenesis. Mater Sci Eng C Mater Biol Appl 124:112041. https://doi.org/10.1016/j.msec.2021.112041

10. Purohit SD, Singh H, Bhaskar R, Yadav I, Chou CF, Gupta MK, Mishra NC (2020) Gelatin-alginate-cerium oxide nanocomposite scaffold for bone regeneration. Mater Sci Eng C Mater Biol Appl 116:111111. https://doi.org/10.1016/j.msec.2020.111111

11. Chen XJ, Shen YS, He MC, Yang F, Yang P, Pang FX, He W, Cao YM, Wei QS (2019) Polydatin promotes the osteogenic differentiation of human bone mesenchymal stem cells by activating the BMP2-Wnt/ $\beta$-catenin signaling pathway. Biomed Pharmacother 112:108746. https://doi.org/10.1016/j.biopha.2019.108746

12. Yuan K, Mei J, Shao D, Zhou F, Qiao H, Liang Y, Li K, Tang T (2020) Cerium oxide nanoparticles regulate osteoclast differentiation bidirectionally by modulating the cellular production of reactive oxygen species. Int J Nanomedicine 15:6355-6372. https:// doi.org/10.2147/IJN.S257741
13. Kizil C, Küchler B, Yan JJ, Özhan G, Moro E, Argenton F, Brand M, Weidinger G, Antos CL (2014) Simplet/Fam53b is required for Wnt signal transduction by regulating $\beta$-catenin nuclear localization. Development 141(18):3529-3539. https://doi.org/10.1242/ dev. 108415

14. Lu B, Zhu DY, Yin JH, Xu H, Zhang CQ, Ke QF, Gao YS, Guo YP (2019) Incorporation of cerium oxide in hollow mesoporous bioglass scaffolds for enhanced bone regeneration by activating the ERK signaling pathway. Biofabrication 11(2):25012. https:// doi.org/10.1088/1758-5090/ab0676

15. Xiang J, Li J, He J, Tang X, Dou C, Cao Z, Yu B, Zhao C, Kang F, Yang L, Dong S, Yang X (2016) Cerium oxide nanoparticle modified scaffold interface enhances vascularization of bone grafts by activating calcium channel of mesenchymal stem cells. ACS Appl Mater Interfaces 8(7):4489-4499. https://doi.org/10.1021/acsami. $6 \mathrm{~b} 00158$

16. Komori $\mathrm{T}$ (2006) Regulation of osteoblast differentiation by transcription factors. J Cell Biochem 99(5):1233-1239. https://doi. org $/ 10.1002 /$ jcb. 20958

17. Komori $\mathrm{T}$ (2018) Runx2, an inducer of osteoblast and chondrocyte differentiation. Histochem Cell Biol 149(4):313-323. https://doi. org/10.1007/s00418-018-1640-6

18. Thermes V, Candal E, Alunni A, Serin G, Bourrat F, Joly JS (2006) Medaka simplet (FAM53B) belongs to a family of novel vertebrate genes controlling cell proliferation. Development 133(10):1881-1890. https://doi.org/10.1242/dev.02350

19. Grafe I, Alexander S, Peterson JR, Snider TN, Levi B, Lee B and Mishina Y (2018) TGF- $\beta$ family signaling in mesenchymal differentiation. Cold Spring Harb Perspect Biol 10(5). https://doi. org/10.1101/cshperspect.a022202

20. Ohba S (2020) Hedgehog signaling in skeletal development: roles of Indian hedgehog and the mode of its action. Int J Mol Sci 21(18). https://doi.org/10.3390/ijms21186665

21. Karner CM, Long F (2017) Wnt signaling and cellular metabolism in osteoblasts. Cell Mol Life Sci 74(9):1649-1657. https://doi.org/ 10.1007/s00018-016-2425-5

22. Maeda K, Kobayashi Y, Koide M, Uehara S, Okamoto M, Ishihara A, Kayama T, Saito M and Marumo K (2019) The regulation of bone metabolism and disorders by Wnt signaling. Int J Mol Sci 20(22). https://doi.org/10.3390/ijms20225525

23. Hong G, He X, Shen Y, Chen X, Yang F, Yang P, Pang F, Han X, He W, Wei Q (2019) Chrysosplenetin promotes osteoblastogenesis of bone marrow stromal cells via $\mathrm{Wnt} / \beta$-catenin pathway and enhances osteogenesis in estrogen deficiency-induced bone loss. Stem Cell Res Ther 10(1):277. https://doi.org/10.1186/ s13287-019-1375-x

Publisher's Note Springer Nature remains neutral with regard to jurisdictional claims in published maps and institutional affiliations. 